\title{
Simulation of Transient Processes in \\ a Generator-Transformer-Load System with Non-Symmetry of Phase Windings \\ of the Stator and Saturation of the Magnetic System
}

\author{
Gleb V. Glazyrin and Nikolay A. Mitrofanov* \\ Novosibirsk State Technical University \\ 20 K. Marks, Novosibirsk, 630073, Russia
}

Received 10.03.2018, received in revised form 27.09.2018, accepted 14.11.2019

\begin{abstract}
Transient processes of a generator-transformer-load system are considered taking into account the saturation of its magnetic system. A method is proposed for numerical simulation of the transient processes of a synchronous machine with the possibility of taking into account the asymmetry of the stator winding and the saturation of the magnetic system. The appearance of asymmetry is possible if the synchronous machine is damaged, in particular, during inter-turn short circuits in the stator winding.

It is based on the direct solution of the differential equations of equilibrium of the emf and voltage drops in the windings in phase coordinates together with the equation of motion of the rotor. In this case, the contour of each phase winding of the stator is described by a separate equation, and different phase parameters can be taken into account.

The mathematical model of the system is realized in the software package MATLAB. The model takes into account one of the possible types of nonsymmetry - an unequal number of turns in phase windings, which makes it possible to simulate interturn short circuits without taking into account the appearance of additional short-circuited circuits.

The model was verified by comparing the transient calculation results obtained using the developed model and using the MATLAB Simulink tools. The simulation results for the idle and load modes are analyzed. The obtained results allowed to draw a conclusion about the adequacy of the realized model.
\end{abstract}

Keywords: synchronous machine, transient process, electric power system, differential equations, numerical method.

Citation: Glazyrin G.V., Mitrofanov N.A. Simulation of transient processes in a generator-transformer-load system with nonsymmetry of phase windings of the stator and saturation of the magnetic system, J. Sib. Fed. Univ. Eng. technol., 2019, 12(8), 939-951. DOI: 10.17516/1999-494X-0193.

(C) Siberian Federal University. All rights reserved

This work is licensed under a Creative Commons Attribution-NonCommercial 4.0 International License (CC BY-NC 4.0).

* Corresponding author E-mail address: n.a.mitrofanov27@gmail.com 


\title{
Моделирование переходных процессов \\ в системе генератор-трансформатор-нагрузка \\ с несимметрией фазных контуров статора
}

\author{
Г.В. Глазырин, Н.А. Митрофанов \\ Новосибирский государственный технический университет \\ Россия, 630073, Новосибирск, пр. Карла Маркса, 20
}

\begin{abstract}
Рассматриваются переходные проиессы в системе генератор-трансформатор-нагрузка. Предложен метод численного моделирования переходных процессов синхронной машины с возможностью учета несимметрии обмотки статора, появление которой возможно при повреждении синхронной машины, в частности при межвитковых коротких замыканиях в обмотке статора.

Метод основан на непосредственном решении дифференциальных уравнений равновесия э.д.с. и падений напряжений в обмотках в фазных координатах совместно с уравнением движения ротора. При этом контур каждой фазной обмотки статора описывают отдельным уравнением, и могут быть учтены отличающиеся параметры фаз.

Математическая модель реализована в программном пакете MATLAB. В модели учтен один из возможных видов несимметрии - неодинаковое число витков в фазных обмотках, что позволяет моделировать межвитковые короткие замыкания без учета появления дополнительных короткозамкнутых контуров.

Выполнена верификация модели посредством сравнения результатов расчета переходных прочессов, полученных при использовании разработанной модели и с помощью средств MATLAB Simulink. Проведен анализ результатов моделирования для режимов: холостого хода и нагрузочного режима. Полученные результаты позволили сделать вывод об адекватности реализованной модели.
\end{abstract}

Ключевые слова: синхронная машина, переходный прочесс, электроэнергетическая система, дифференциальные уравнения, численный метод.

\section{Введение}

Известно, что аналитическое исследование электромагнитных и электромеханических переходных процессов в синхронной машине с учетом всех влияющих факторов является весьма сложной задачей. В связи с этим для упрощения расчета приходится делать ряд допущений, которые вносят некоторые погрешности в оценку рассматриваемых параметров синхронной машины. Практическими методами расчета переходных процессов синхронной машины активно занимаются В.Ф. Сивокобыленко [1] (расчет синхронной машины при учете ротора машины многоконтурными схемами) и С.А. Харитонов [2] (описание электромагнитных переходных процессов в системах генерирования электрической энергии для автономных объектов). К основным допущениям, применяемым в таких методах расчета, можно отнести следующие:

- магнитная система машины не насыщена, в результате чего индуктивности машины не зависят от намагничивающей силы;

- вместо действительных кривых распределения магнитной индукции в воздушном зазоре по расточке статора учитываются только их составляющие первой гармоники; 
- в магнитной системе машины отсутствуют какие-либо потери;

- считается, что конструкция машины обеспечивает полную симметрию фазных обмоток статора. Ротор также симметричен относительно своих продольной и поперечной осей [3].

Однако в некоторых случаях принятые при расчете допущения не позволяют выявить и провести корректный анализ ненормальных режимов синхронной машины. К такому режиму, например, можно отнести несимметрию фазных обмоток статора, вызванную наличием витковых замыканий в одной из них.

Существующие программные пакеты моделирования переходных процессов в электроэнергетических системах, такие как MATLAB Simulink [4], PSCAD, Mustang, используют для описания электромагнитных процессов синхронной машины уравнения Парка-Горева [3], которые записываются в неподвижной относительно ротора системе координат и предполагают полную симметрию фазных обмоток статора. Очевидно, что такой метод моделирования не может быть применен для расчета процессов в поврежденной синхронной машине с отличающимися параметрами фазных обмоток.

В исследуемой модели синхронной машины учитывают по одному продольному и поперечному демпферному контуру, пренебрегая влиянием эффекта вытеснения токов. В рамках же поставленных в исследовании задач допущение последнего не приведет к потере точности и недостоверным результатам.

В статье приведен вывод системы дифференциальных уравнений, описывающих переходные процессы трехфазной синхронной машины с учетом индивидуальных активных сопротивлений и индуктивностей обмоток. Далее произведено сравнение результатов моделирования переходных процессов в системе, выполненного посредством библиотеки блоков SimPowerSystems, входящей в состав среды MATLAB Simulink, с результатами, полученными с помощью предложенного метода.

\section{Исходные уравнения, характеризующие работу системы}

Рассмотрим синхронную машину, имеющую три фазных обмотки, контур возбуждения, а также одну продольную и одну поперечную демпферные обмотки.

Обозначим через $u_{\eta}(\eta=a, b, c)$ и $u_{f}$ мгновенные значения напряжений на фазных обмотках и обмотке возбуждения соответственно; $i_{\eta}$ и $i_{f}$ - мгновенные значения токов; $\psi_{\eta}$ и $\psi_{f}-$ результирующие потокосцепления обмоток; $R_{\eta}$ и $R_{f}$ - активные сопротивления фазных обмоток и обмотки возбуждения. Тогда дифференциальные уравнения равновесия э.д.с. и падений напряжений в контурах синхронной машины будут иметь вид [3]

$$
\left\{\begin{array}{l}
u_{\eta}=-\frac{d \psi_{\eta}}{d t}-R_{\eta} i_{\eta} \quad(\eta=a, b, c) ; \\
u_{f}=\frac{d \psi_{f}}{d t}+R_{f} i_{f} .
\end{array}\right.
$$

Кроме того, систему дифференциальных уравнений (1) следует дополнить уравнениями равновесия э.д.с. и падений напряжений в демпферных контурах:

$$
-941-
$$




$$
\left\{\begin{array}{l}
0=-\frac{d \psi_{\mathrm{y} d}}{d t}-R_{\mathrm{y} d} i_{\mathrm{y} d} ; \\
0=-\frac{d \psi_{\mathrm{y} q}}{d t}-R_{\mathrm{y} q} i_{\mathrm{yq}},
\end{array}\right.
$$

где $\psi_{y d}$ и $\psi_{y q}$ - результирующие потокосцепления продольной и поперечной демпферных обмоток соответственно; $R_{y d}$ и $R_{y q}-$ их активные сопротивления; $i_{y d}$ и $i_{y q}-$ мгновенные значения токов в демпферных контурах.

Предлагаемый метод расчета переходных процессов основан на совместном решении уравнений (1) и (2), дополненных выражениями падений напряжений на сопротивлениях трансформатора и нагрузки. Такой подход делает возможным моделирование синхронной машины с различными параметрами фазных обмоток за счет описания электромагнитных процессов в каждой фазе отдельным дифференциальным уравнением.

\section{Вывод дифференциальных уравнений}

\section{для системы генератор-трансформатор-нагрузка}

Наиболее простая результирующая система дифференциальных уравнений получается в случае подключения нагрузки по схеме «звезда» с нулевым проводом, не имеющим сопротив-



В действительности схемы выдачи мощности электростанций предусматривают работу генераторов с изолированной нейтралью (без нулевого провода). Как правило, генератор подключается к обмотке повышающего трансформатора, соединенной в «треугольник». Для точного расчета переходных процессов в таких схемах необходимо моделирование как генератора, так и трансформатора, что рассматривается в статье. Схема представлена на рис. 1.

При расчете переходных процессов синхронной машины, работающей на автономную активно-индуктивную нагрузку, необходимо учесть следующие преобразования, в которых $u_{k}(k=a b, b c, c a)$ - мгновенные значения линейных напряжений на выводах синхронной машины; $i_{k}-$ мгновенные значения линейных токов:

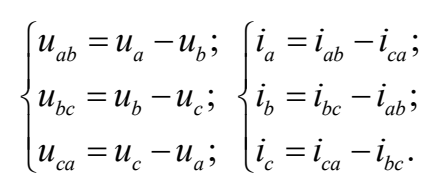

Тогда уравнения равновесия э.д.с. и падений напряжений в контурах синхронной машины в блоке с повышающим трансформатором (1) будут определяться как

$$
\left\{\begin{array}{c}
u_{a b T .1}=\left(L_{a b T .1}+L_{a b \sigma T .1}\right) \frac{d i_{a b .1}}{d t}-M_{b T .1}\left[\frac{M_{b T .1}}{L_{\sum b .2}} \frac{d i_{a b .1}}{d t}-\frac{\left(R_{b n g .2}+R_{b T .2}\right)}{L_{\sum b .2}} i_{b .2}\right]+R_{a b T .1} i_{a b .1} \\
u_{b c T .1}=\left(L_{b c T .1}+L_{b c \sigma T .1}\right) \frac{d i_{b c .1}}{d t}-M_{c T .1}\left[\frac{M_{c T .1}}{L_{\sum c .2}} \frac{d i_{b c .1}}{d t}-\frac{\left(R_{c n g .2}+R_{c T .2}\right)}{L_{\sum c .2}} i_{c .2}\right]+R_{b c T .1} i_{b c .1} \\
u_{c a T .1}=\left(L_{c a T .1}+L_{c a \sigma T .1}\right) \frac{d i_{c a .1}}{d t}-M_{a T .1}\left[\frac{M_{a T .1}}{L_{\sum a .2}} \frac{d i_{c a .1}}{d t}-\frac{\left(R_{a n g .2}+R_{a T .2}\right)}{L_{\sum a .2}} i_{a .2}\right]+R_{c a T .1} i_{c a .1} \\
-942-
\end{array}\right.
$$




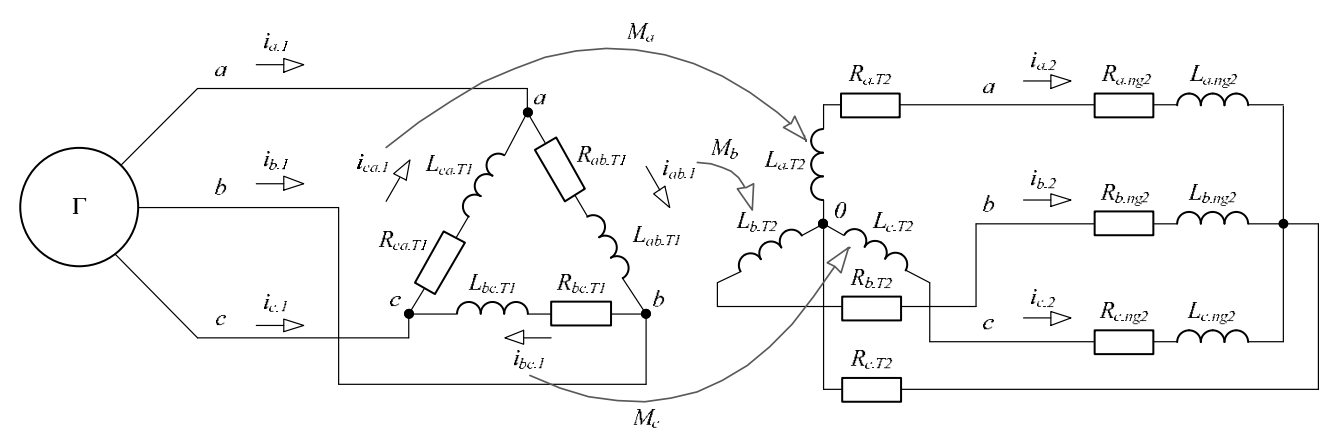

Рис. 1. Схема подключения нагрузки

Fig. 1. Load connection scheme

где $L_{k T .1}, L_{k \sigma T .1}(k=a b, b c, c a)$ - собственное значение индуктивности первичной обмотки трансформатора, обусловленное основным магнитным потоком и потоком рассеяния соответственно; $M_{\eta T}(\eta=a, b, c)$ - взаимная индуктивность обмоток трансформатора; $L_{\sum \eta .2}=L_{\eta n g .2}+L_{\eta T .2}+L_{\eta \sigma T .2}-$ суммарное значение индуктивностей на вторичной стороне системы; $R_{\eta n g .2}-$ активное сопротивление нагрузки; $R_{k T .1}, R_{\eta T .2}$ - активное сопротивление первичной и вторичной обмотки трансформатора; $u_{k T .1}$ - мгновенные значения линейных напряжений на первичной обмотке трансформатора, $\psi_{i j}=\psi_{i}-\psi_{j}$.

Обращаясь к описанию электромагнитных процессов синхронной машины уравнения Парка-Горева, которые записывают в неподвижной относительно ротора системе координат, видис, что продольное синхронное индуктивное сопротивление не является постоянным, а зависит от насыщения машины. Эта зависимость приближенно может быть выявлена с помощью характеристики X.х. машины. Подробно определение влияния насыщения магнитной системы на величину синхронного индуктивного сопротивления изложено в [6], где рассмотрено построение характеристики х.х. и выведены все необходимые величины. В данном исследовании насыщение магнитной системы как синхронной машины, так и повышающего трансформатора не учитывается.

Стоит отметить, что в исследовании учтены только взаимные индуктивности между первичной и вторичной обмотками трансформатора. Взаимные индуктивности между фазами не принимают во внимание. Таким образом, модель можно представить в виде группы однофазных трансформаторов.

При расчете переходных процессов синхронной машины, работающей на автономную активно-индуктивную нагрузку, дифференциальные уравнения принимают вид 


$$
\left\{\begin{array}{l}
\frac{d \psi_{a b}}{d t}=-u_{a b T .1}-\left[\left(R_{g . a}+R_{g . b}\right) i_{a b}-R_{g . a} i_{c a}-R_{g . b} i_{b c}\right] \\
\frac{d \psi_{b c}}{d t}=-u_{b c T .1}-\left[\left(R_{g . b}+R_{g . c}\right) i_{b c}-R_{g . b} i_{a b}-R_{g . c} i_{c a}\right] \\
\frac{d \psi_{c a}}{d t}=-u_{c a T .1}-\left[\left(R_{g . c}+R_{g . a}\right) i_{c a}-R_{g . c} i_{b c}-R_{g . a} i_{a b}\right] \\
\frac{d \psi_{f}}{d t}=u_{f}-R_{f} i_{f} \\
\frac{d \psi_{\mathrm{y} d}}{d t}=-R_{\mathrm{y} d} i_{\mathrm{y} d} \\
\frac{d \psi_{\mathrm{y} q}}{d t}=-R_{\mathrm{y} q} i_{\mathrm{y} q}
\end{array}\right.
$$

где $K_{g . \eta}(\eta=a, b, c)$ - активное сопротивление контура фазной обмотки генератора.

Для упрощения восприятия условно примем замену переменных, где $L_{k \Sigma}(k=a b, b c, c a)-$ суммарные собственные индуктивности контуров; $M_{k . e}$ - эквивалентные взаимные индуктивности контуров; $u_{\Sigma k}-$ мгновенные значения падений напряжения на активных сопротивлениях контуров:

$$
\left\{\begin{array}{l}
\left\{\begin{array}{l}
L_{a b \Sigma}=L_{a}+L_{b}-2 M_{a b}+L_{a b T .1}+L_{a b \sigma T .1}+\frac{M_{b T .1}^{2}}{L_{\sum b .2}} \\
L_{b c \Sigma}=L_{b}+L_{c}-2 M_{b c}+L_{b c T .1}+L_{b c \sigma T .1}+\frac{M_{c T .1}^{2}}{L_{\sum c .2}} \\
L_{c a \Sigma}=L_{c}+L_{a}-2 M_{c a}+L_{c a T .1}+L_{c a \sigma T .1}+\frac{M_{a T .1}^{2}}{L_{\sum a .2}} ; \\
M_{a b . e}=M_{b c}+M_{a b}-M_{c a}-L_{b} ; \\
M_{b c . e}=M_{b c}+M_{c a}-M_{a b}-L_{c} ; \\
M_{c a . e}=M_{c a}+M_{a b}-M_{b c}-L_{a} ; \\
u_{\sum a b}=-M_{b T .1} \frac{\left(R_{b n g .2}+R_{b T .2}\right)}{L_{\sum b .2}} i_{b .2}-\left(R_{g . a}+R_{g . b}+R_{a b .1}\right) i_{a b}+R_{g . a} i_{c a}+R_{g . b} i_{b c} ; \\
u_{\sum b c}=-M_{c T .1} \frac{\left(R_{c n g .2}+R_{c T .2}\right)}{L_{\sum c .2}} i_{c .2}-\left(R_{g . b}+R_{g . c}+R_{b c .1}\right) i_{b c}+R_{g . b} i_{a b}+R_{g . c} i_{c a} ; \\
u_{\sum c a}=-M_{a T .1} \frac{\left(R_{a n g .2}+R_{a T .2}\right)}{L_{\sum a .2}} i_{a .2}-\left(R_{g . c}+R_{g . a}+R_{c a .1}\right) i_{c a}+R_{g . c} i_{b c}+R_{g . a} i_{a b} .
\end{array}\right.
\end{array}\right.
$$

Коэффициенты пропорциональности $L_{a}, L_{b}, L_{c}, L_{f}, L_{y d}, L_{y q}$ есть собственные индуктивности фазных обмоток, обмотки возбуждения, продольной и поперечной демпферных обмоток соответственно, символом $M$ обозначены взаимные индуктивности обмоток. Как определяются эти коэффициенты, подробно раскрыто в [3], и останавливаться на их рассмотрении не имеет смысла. Стоит лишь отметить, что большинство индуктивностей, входящих в выражение (5), являются функциями угла поворота ротора $\gamma$. При нарушении симметрии фазных контуров статора эти индуктивности могут быть определены с помощью дополнительного коэффициента, учитывающего долю замкнувшихся витков $k_{w \eta}(\eta=a, b, c)$. 
Решение системы уравнений (1) включает в себя определение производной $d \psi_{k} / d t$ от сложной функции, зависящей от токов в контурах и угла поворота ротора. Для этого к системе (2) применим формулу производной сложной функции

$$
\frac{d \psi_{k}}{d t}=\frac{\partial \psi_{k}}{\partial \gamma} \frac{d \gamma}{d t}+\sum_{m} \frac{\partial \psi_{m}}{\partial i_{m}} \frac{d i_{m}}{d t}(k, m=a b, b c, c a, f, \mathrm{y} d, \mathrm{y} q)
$$

и подставим полученные выражения в (1). Так как процесс определения производных для разных контуров однотипен, рассмотрим лишь одно уравнение для контура “ $a b$ ” статора:

$$
\begin{aligned}
& \frac{d \psi_{a b}}{d t}=\frac{\partial \psi_{a b}}{\partial \gamma} \omega+\frac{d i_{a b}}{d t} L_{a b . e}+\frac{d i_{b c}}{d t} M_{. a b . e}+\frac{d i_{c a}}{d t} M_{c a . e}+\left(M_{a f}-M_{b f}\right) \frac{d i_{f}}{d t}+ \\
& +\left(M_{a y d}-M_{b y d}\right) \frac{d i_{\mathrm{y} d}}{d t}+\left(M_{a y q}-M_{b y q}\right) \frac{d i_{\mathrm{yq}}}{d t}=-L_{\mathrm{n} . a b} \frac{d i_{a b}}{d t}-u_{\sum a b} .
\end{aligned}
$$

Уравнения для остальных контуров могут быть получены тем же способом.

Частную производную потокосцепления по углу, входящую в (6), выразим следующим образом:

$$
\begin{aligned}
& \frac{\partial \psi_{a b}}{\partial \gamma}=\frac{d L_{a b . e}}{d \gamma} i_{a b}+\frac{d M_{. a b . e}}{d \gamma} i_{b c}+\frac{d M_{c a . e}}{d \gamma} i_{c a}+\frac{d\left(M_{a f}-M_{b f}\right)}{d \gamma} i_{f}+ \\
& +\frac{d\left(M_{a y d}-M_{b y d}\right)}{d \gamma} i_{\mathrm{y} d}+\frac{d\left(M_{a y q}-M_{b y q}\right)}{d \gamma} i_{y q} .
\end{aligned}
$$

Выведенные в (6) производные индуктивностей по углу можно определить, зная исходные выражения индуктивностей, полученные в [3].

В результате подстановки (7) в (6) получим уравнение, связывающее функции времени токи в обмотках и угол поворота ротора - и производные этих функций.

При рассмотрении всех контуров синхронной машины вместо одного уравнения (6) получим следующую систему уравнений в матричном виде:

$$
\left[\begin{array}{cccccc}
L_{a b \Sigma} & M_{. a b . e} & M_{c a . e} & M_{a b f} & M_{a b y d} & M_{a b y q} \\
M_{. a b . e} & L_{b c \Sigma} & M_{b c . e} & M_{b c f} & M_{b c y d} & M_{b c y q} \\
M_{c a . e} & M_{b c . e} & L_{c a \Sigma} & M_{c a f} & M_{c a y d} & M_{c a y q} \\
M_{a b f} & M_{b c f} & M_{c a f} & L_{f} & M_{f y d} & 0 \\
M_{a b y d} & M_{b c y d} & M_{c a y d} & M_{f y d} & L_{\mathrm{y} d} & 0 \\
M_{a b y q} & M_{b c y q} & M_{c a y q} & 0 & 0 & L_{\mathrm{yq}}
\end{array}\right] \cdot\left[\begin{array}{c}
d i_{a b} / d t \\
d i_{b c} / d t \\
d i_{c a} / d t \\
d i_{f} / d t \\
d i_{\mathrm{yd}} / d t \\
d i_{\mathrm{yq}} / d t
\end{array}\right]=\left[\begin{array}{c}
-\left(\partial \psi_{a b} / \partial \gamma\right) \omega+u_{\sum a b} \\
-\left(\partial \psi_{b c} / \partial \gamma\right) \omega+u_{\Sigma b c} \\
-\left(\partial \psi_{c a} / \partial \gamma\right) \omega+u_{\sum c a} \\
-\left(\partial \psi_{f} / \partial \gamma\right) \omega-R_{f} i_{f}+u_{f} \\
-\left(\partial \psi_{\mathrm{y} d} / \partial \gamma\right) \omega-R_{\mathrm{yd}} i_{\mathrm{y} d} \\
-\left(\partial \psi_{\mathrm{yq}} / \partial \gamma\right) \omega-R_{\mathrm{yy} i_{\mathrm{y} q}}
\end{array}\right],
$$

где $M_{k f}, M_{k y d}, M_{k y q}(k=a b, b c, c a)$ - значения взаимной индуктивности обмотки возбуждения, продольной и поперечной демпферных обмоток.

Помимо системы (8), необходимо определить значения токов во вторичной обмотке трансформатора, для чего воспользуемся системой уравнений: 


$$
\left\{\begin{array}{l}
\frac{d i_{a .2}}{d t}=\frac{M_{a T .1}}{L_{\sum a .2}} \frac{d i_{c a .1}}{d t}-\frac{\left(R_{a n g .2}+R_{a T .2}\right)}{L_{\sum a .2}} i_{a .2} \\
\frac{d i_{b .2}}{d t}=\frac{M_{b T .1}}{L_{\sum b .2}} \frac{d i_{a b .1}}{d t}-\frac{\left(R_{b n g .2}+R_{b T .2}\right)}{L_{\sum b .2}} i_{b .2} \\
\frac{d i_{c .2}}{d t}=\frac{M_{c T .1}}{L_{\sum c .2}} \frac{d i_{b c .1}}{d t}-\frac{\left(R_{c n g .2}+R_{c T .2}\right)}{L_{\sum c .2}} i_{c .2}
\end{array}\right.
$$

Полученная система уравнений (8) позволяет определять производные токов в обмотках по известным значениям функций (токов, угла поворота ротора и частоты).

Для описания электромеханических процессов синхронной машины применим дифференциальное уравнение движения ротора в форме д’Аламбера [3]:

$$
M_{\mathrm{T}}-J \frac{d \omega}{d t}+\frac{\partial W_{\mathrm{M}}}{\partial \gamma}=0
$$

где $M_{\mathrm{T}}$ - момент турбины; $J$ - момент инерции ротора; $W_{\mathrm{M}}$ - энергия магнитных полей машины, которая может быть определена по формуле [3]:

$$
W_{\mathrm{M}}=\frac{1}{2} \sum_{k} i_{k} \psi_{k}, \quad k=a, b, c, f, \mathrm{y} d, \mathrm{y} q .
$$

При подстановке (10) в (9) и преобразований получим следующее выражение для расчета производной частоты по известным значениям функций:

$$
\frac{d \omega}{d t}=\frac{P_{\mathrm{T}}}{J \omega}+\frac{1}{2 J}\left(i_{a} \frac{\partial \psi_{a}}{\partial \gamma}+i_{b} \frac{\partial \psi_{b}}{\partial \gamma}+i_{c} \frac{\partial \psi_{c}}{\partial \gamma}+i_{f} \frac{\partial \psi_{f}}{\partial \gamma}+i_{\mathrm{y} d} \frac{\partial \psi_{\mathrm{y} d}}{\partial \gamma}+i_{\mathrm{y} q} \frac{\partial \psi_{\mathrm{y} q}}{\partial \gamma}\right),
$$

где $P_{\mathrm{T}}$ - мощность турбины. Частные производные потокосцеплений по углу, полученные в (11), входят также в систему уравнений (8), и их определение уже рассмотрено ранее.

Для получения полной системы дифференциальных уравнений, описывающих переходные процессы синхронной машины, необходимо (8) и (11) дополнить связью между угловой частотой и углом поворота ротора:

$$
\frac{d \gamma}{d t}=\omega
$$

\section{Описание метода численного моделирования}

Численное решение системы обыкновенных дифференциальных уравнений (разностными методами) требует расчета производных функций по известным значениям самих функций на каждом шаге интегрирования [7]. Для этого система обычно представляется в форме Коши. В рассматриваемом случае два уравнения (11) и (12) содержат по одной производной в левой части, в то время как оставшиеся уравнения заданы в матричном виде (9), причем разделение переменных затруднительно из-за большого порядка матриц.

Кроме того, указанные уравнения имеют переменные коэффициенты, зависящие от угла поворота ротора $\gamma$. В итоге требуется пересчет большинства коэффициентов на каждом шаге 
интегрирования, и представление (8) в явной форме Коши не обеспечило бы существенного снижения объема вычислений.

Предложенная математическая модель синхронного генератора реализована при помощи программного пакета MATLAB. Данная программа широко применяется в различного рода исследованиях переходных процессов в синхронных и асинхронных машинах [8-11]. Для решения системы дифференциальных уравнений использована функция оde 45 , основанная на одношаговом явном методе Рунге-Кутта 4-го и 5-го порядка [4]. За основу метода расчета производных при численном решении системы дифференциальных уравнений взят алгоритм, предложенный в [5].

Однако не всегда применение пакета MATLAB отвечает требуемому быстродействию. Для повышения скорости вычислений авторы $[5,12]$ предлагают методы дополнительной оптимизации программного кода.

\section{Верификация реализуемой модели}

В качестве примера для сравнения моделей выполнены расчеты переходных процессов генератора ТВВ-200-2АУ3 с полной симметрией фазных обмоток статора и следующими параметрами: $S_{\text {ном }}=235.3 \mathrm{MB} \cdot \mathrm{A}, U_{\text {ном }}=15.57 \mathrm{\kappa B}, f_{\text {ном }}=50$ Гц, $x_{d}=2.106, x_{d}^{\prime}=0,272, x_{d}^{\prime \prime}=0,1805$, $x_{q}=2.106, x_{q}^{\prime \prime}=0.1805, x_{\sigma}=0.166, T_{d}^{\prime}=0.91 \mathrm{c}, T_{d}^{\prime \prime}=0.114 \mathrm{c}, T_{q}^{\prime \prime}=0.114 \mathrm{c}$, активное сопротивление обмотки статора $R_{s}=0.00152$ Ом. Трансформатор ТДЦ-250000/110 имеет параметры: $S_{\text {номт }}=250 \mathrm{MB} \cdot \mathrm{A}, U_{\text {номвн }}=121 \kappa \mathrm{B}, U_{\text {номнН }}=15.75 \kappa \mathrm{B}, U_{k}=10.5 \%, P_{k}=640$ кВт, $I_{k}=0.5 \%$.

Работа системы рассматривается в нескольких режимах: в нормальном установившемся режиме под нагрузкой, подача напряжения на обмотку возбуждения при отключенной нагрузке.

Результаты расчетов в нормальном установившемся режиме под нагрузкой с помощью моделирования численным методом и в среде MATLAB Simulink приведены на рис. 2 и 3.

Из сравнения полученных результатов нормального установившегося режима работы генератора можно увидеть, что при численном методе моделирования имеем следующее: амплитуд-
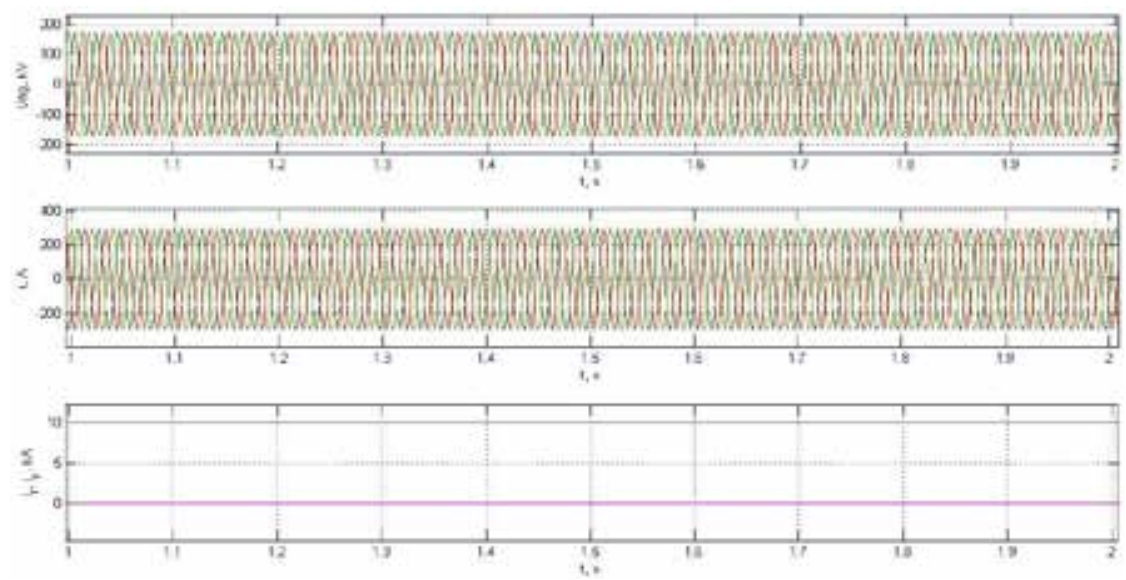

Рис. 2. Результаты расчетов численным методом моделирования в нормальном установившемся режиме под нагрузкой

Fig. 2. The results of calculations by the numerical simulation method in the normal steady state under load

$$
-947-
$$




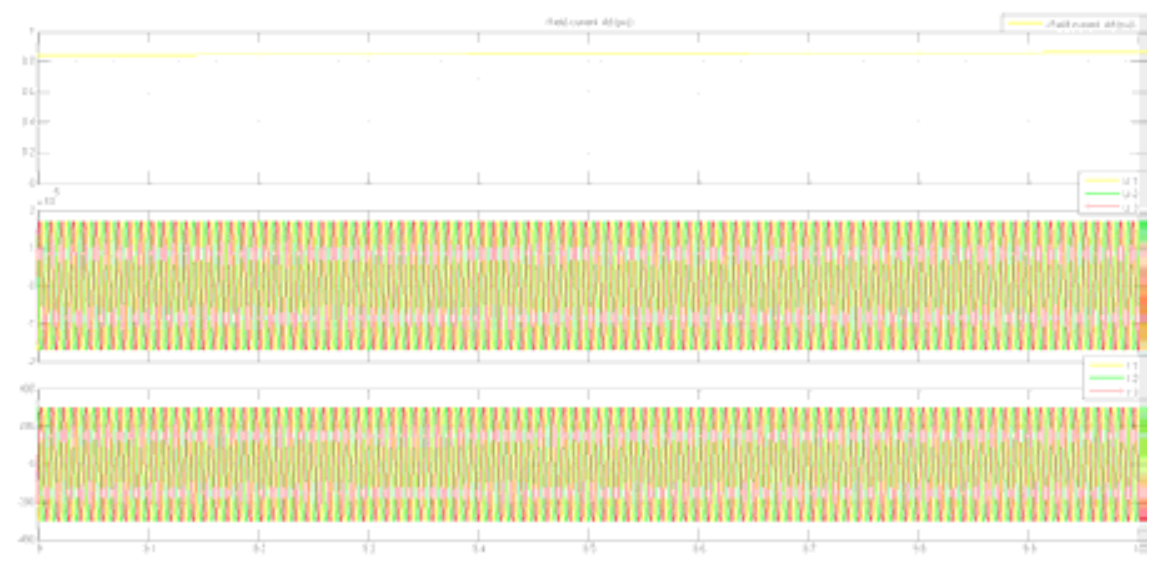

Рис. 3. Результаты расчетов в среде Simulink в нормальном установившемся режиме под нагрузкой

Fig. 3. Results of Simulink calculations in the normal steady state under load

ное значение напряжения на вторичной обмотке трансформатора - составляет $u_{a b T .2}=172.7$ кВ, амплитудное значение фазного тока вторичной обмотки трансформатора $i_{a .2}=294$ А. При моделировании генератора в среде MATLAB Simulink эти значения составляют $u_{a b T .2}=176.1 \mathrm{\kappa B}$, $i_{a .2}=302$ А соответственно.

Результаты расчетов при подаче напряжения на обмотку возбуждения и отключенной нагрузке с помощью моделирования численным методом и в среде Simulink приведены на рис. 4 и 5.

Сравнение полученных результатов режима холостого хода генератора показывает, что при численном методе моделирования амплитудное значение э.д.с. контуров статора составляет $u_{a b T .2}=173.1$ кB. При моделировании генератора в среде MATLAB Simulink $u_{a b T .2}=175.2$ кB.
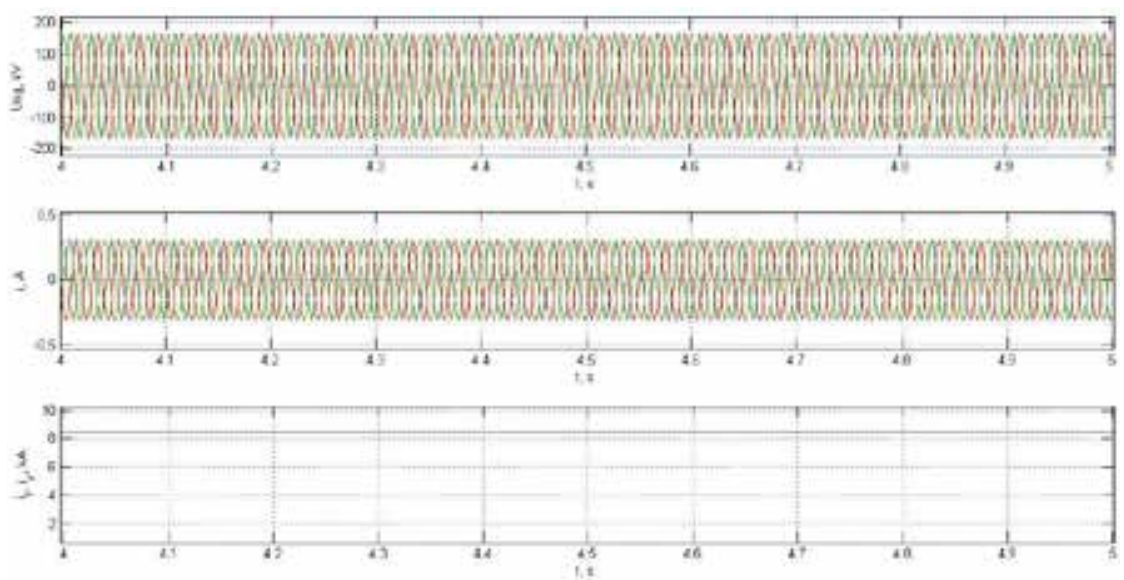

Рис. 4. Результаты расчетов численным методом моделирования при подаче напряжения на обмотку возбуждения и отключенной нагрузке

Fig. 4. The results of calculations by a numerical simulation method with a voltage applied to the excitation winding and the disconnected load 


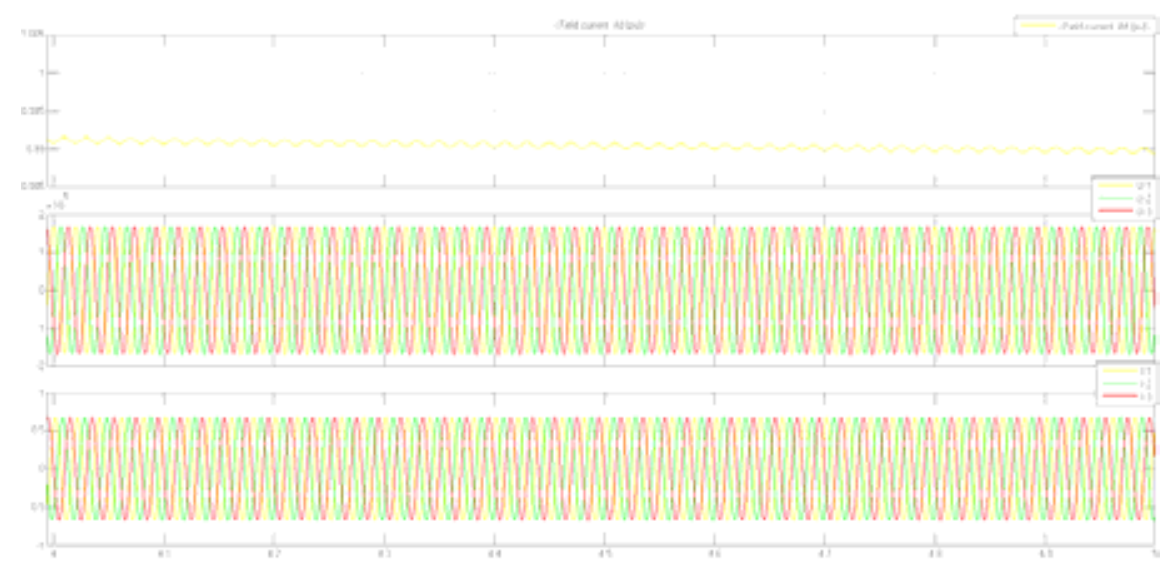

Рис. 5. Результаты расчетов в среде Simulink при подаче напряжения на обмотку возбуждения и отключенной нагрузке

Fig. 5. Results of calculations in the Simulink environment when applying voltage to the excitation winding and the disconnected load
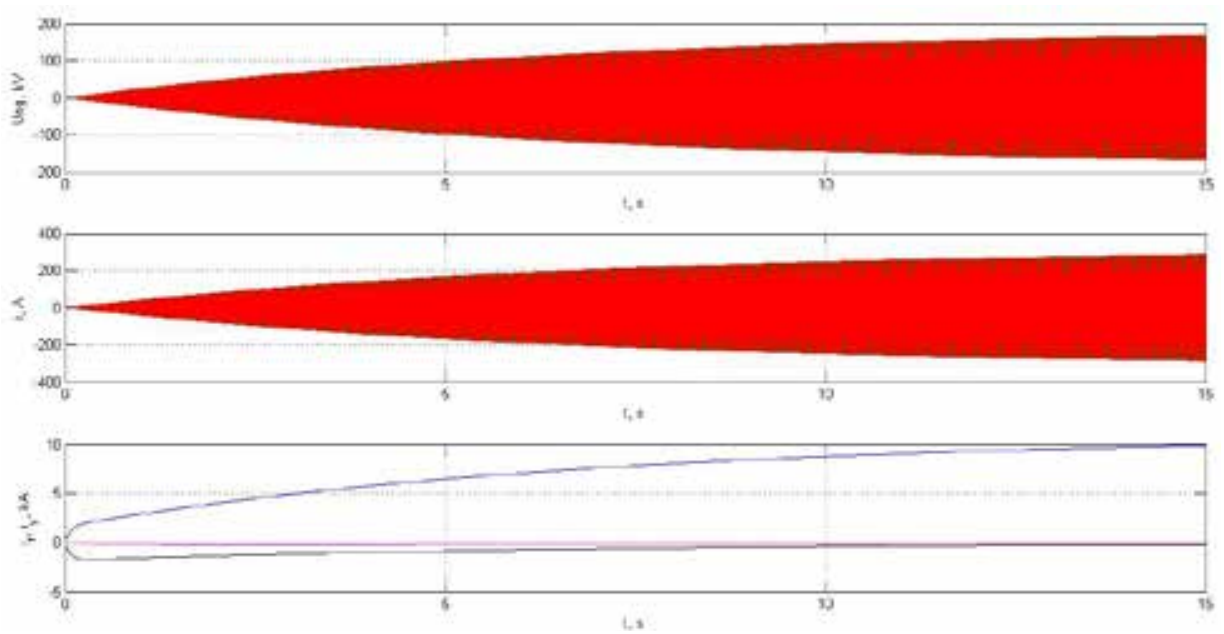

Рис. 6. Результаты расчетов численным методом моделирования переходного процесса в синхронной машине

Fig. 6. Results of numerical simulation of the transient process in a synchronous machine

На рис. 6 представлены результаты численного моделирования переходного процесса в синхронной машине при выходе ее на нормальный установившийся режим работы.

\section{Выводы}

Полученные в ходе исследования результаты сравнения расчетного метода и модели в среде MATLAB Simulink при работе с симметричными фазными контурами статора и без учета насыщения магнитной системы показали достоверность предлагаемого численного метода моделирования. 
Реализованная математическая модель дает возможность рассматривать влияние несимметрии фазных контуров статора и насыщения магнитной системы при моделировании синхронной машины.

В перспективе предлагаемый метод позволит использовать результаты расчетов переходных процессов синхронной машины для анализа работы и создания новых алгоритмов релейной защиты генераторов электростанций.

\section{Список литературы}

[1] Сивокобыленко В.Ф. Математическое моделирование синхронной машины с многоконтурным ротором в фазных координатах. ISSN 1607-7970. Техническая электродинамика, 2015, 1, 51-58 [Sivokobyilenko V.F. Mathematical modeling of a synchronous machine with a multi-loop rotor in phase coordinates. ISSN 1607-7970. Technical electrodynamics, 2015, 1, 51-58 (in Russian)]

[2] Харитонов С.А. Электромагнитные переходные прочессы в системах генерирования электрической энергии для автономных объектов. Новосибирск: Изд-во НГТУ, 2011. 536 с. [Haritonov S.A. Processes in Power Generating Systems for Stand-Alone Units. Novosibirsk, NSTU, 2011. 536 p. (in Russian)]

[3] Горев А.А. Переходные прочессы синхронной машины. Л.: Наука, 1985. 502 с. [Gorev A.A. Transient processes of a synchronous machine. L., Science, 1985. 502 p. (in Russian)]

[4] Черных И.В. Моделирование электротехнических устройств в MATLAB. SimPowerSystems u Simulink. М.: ДМК Пресc, 2008. 288 c. [Chernyih I.V. Modeling of electrical devices in MATLAB. SimPowerSystems and Simulink. M., HMC Press , 2008. 288 p. (in Russian)]

[5] Глазырин Г.В. Моделирование переходных процессов синхронной машины с несимметрией фазных обмоток статора. Вестник МЭИ, 2017, 5, 34-39 [Glazyirin G.V. Simulation of transient processes of a synchronous machine with the asymmetry of phase stator windings. Newsletter MEI, 2017, 5, 34-39 (in Russian)]

[6] Жданов П.С. Вопросы устойчивости электрических систем. М.: Энергия, 1979. 456 с. [Zhdanov P.S. Questions of the stability of electrical systems. M., Energy, 1979. 456 p. (in Russian)]

[7] Мэтьюз Д.Г., Финк К.Д. Численные методы. Использование MATLAB, 3-е изд: Пер. с англ. М.: Издательский дом «Вильямс», 2001. 720 c. [Mathews D.G., Finck K.D. Numerical methods. Using MATLAB, 3rd edition. M., Publishing house «Williams», 2001. 720 p. (in Russian)]

[8] Файзиев М.М., Курбонов Н.А., Имамназаров А.Б., Бекишев А.Э. Моделирование пуска асинхронных двигателей в МАTLAB. Вестник науки и образования, 2017, 3(27), 4247 [Fayziev M.M., Kurbonov N.A., Imamnazarov A.B., Bekishev A.E. Modeling of start-up of asynchronous motors in MATLAB, Bulletin of Science and Education, 2017, 3(27), 42-47 (in Russian)]

[9] Субботина В.А., Тюленев M.E. Simulink - модель для исследования пуска синхронного двигателя при пониженном напряжении. Электротехника, информационные технологии, системы управления, 2014, 11, 102-109 [Subbotina V.A., Tyulenev M.E. Simulink is a model for investigating the start-up of a synchronous motor under reduced voltage. Electrical engineering, information technology, control systems, 2014, 11, 102-109 (in Russian)]

[10] Федий К.С., Встовский С.А., Полошков Н.Е. Моделирование переходных процессов в торцевом синхронном генераторе в пакете MATLAB. Журнал Сибирского федерального университета. Техника и технологии, 2017, 10(5), 691-698 [Fediy K.S., Vstovsky S.A., Poloshkov N.E. 
Simulation of transients in the face synchronous generator in the MATLAB package, Journal of Siberian Federal University. Engineering and technology, 2017, 10(5), 691-698 (in Russian)]

[11] Demiroren A. and Zeynelgil H.L. Modelling and simulation of synchronous machine transient analysis using SIMULINK. International Journal of Electrical Engineering Education, 2002, 39/4, $337-346$.

[12] Hafnaoui I., Ayari R., Nicolescu G., Beltrame G. A simulation-based model generator for software performance estimation. SCSC '16 Proceedings of the Summer Computer Simulation Conference. USA, 2016, ISBN: 978-1-5108-2424-9. 